\title{
Differences in Clinical and Demographic Characteristics in Subarachnoid Hemorrhage Patients with and Without an Aneurysm: A Cross-Sectional Study in Western Iran
}

\author{
Mojtaba Khazaei ${ }^{1,}$, , Poua Bahari ${ }^{2}$, Shahir Mazaheri ${ }^{1}$ and Abbas Moradi ${ }^{3}$ \\ ${ }^{1}$ Department of Neurology, Sina (Farshchian) Educational and Medical Center, School of Medicine, Hamadan University of Medical Sciences, Hamadan, Iran \\ ${ }^{2}$ Student Research Committee, Hamadan University of Medical Sciences, Hamadan, Iran \\ ${ }^{3}$ Department of Community Medicine, Hamadan University of Medical Sciences, Hamadan, Iran \\ Corresponding author: Department of Neurology, Sina (Farshchian) Educational and Medical Center, School of Medicine, Hamadan University of Medical Sciences, Hamadan, \\ Iran. Email: mojtaba.khazaei2361@gmail.com
}

Received 2019 October 16; Revised 2020 March 03; Accepted 2020 March 03.

\begin{abstract}
Background: Subarachnoid hemorrhage (SAH) is one of the most costly and important causes of death and disability worldwide. Aneurysmal type of this hemorrhage has high fatality and permanent disability rates.

Objectives: In the present study, demographic and clinical characteristics of SAH with and without an aneurysm were compared. Methods: In this cross-sectional study, via the census method, all patients diagnosed with subarachnoid hemorrhage who were admitted to Farshchian Hospital in Hamadan during 2015 - 2016 were assessed. For SAH patients, both with and without an aneurysm, we used medical records to obtain demographic and clinical information. Data was analyzed using SPSS software version 21 , at a $95 \%$ confidence level.

Results: Overall, the medical records of 69 patients were investigated in this study. Among them, 17 (24.63\%) had an aneurysm. There was a significant difference between the age of SAH patients and aneurysm $(\mathrm{P}=0.003)$. Concerning patient risk factors, $59.4 \%$ had a history of hypertension, $29 \%$ had a history of smoking, and 17.4\% had a history of diabetes. These risk factors and patient aneurysm status were not significantly correlated $(\mathrm{P}>0.05)$. The proportion of death, persistent defect, hydrocephaly, and re-bleeding in investigated patients was $36.23 \%, 43.48 \%, 26.09 \%$, and $17.39 \%$, respectively.

Conclusions: According to the findings, hypertension and smoking were two of the common modifiable risk factors in aneurysmal and non-aneurysmal SAH patients. Unlike non-aneurysmal SAH, aneurysmal SAH was more common in younger patients.
\end{abstract}

Keywords: Stroke, Subarachnoid Hemorrhage, Aneurysm

\section{Background}

Stroke is one of the most expensive and important causes of disability-adjusted life year (DALYs) (1). Approximately $5 \%$ of strokes are caused by subarachnoid hemorrhage (SAH) following the rupture of an aneurysm that primarily occurs at an early age and is associated with a poor prognosis (2).

Compared to other cerebral hemorrhages, SAH occurs at an early age, as well as in the sixth decade of life (3). Gender and country of residence have a significant effect on the incidence of SAH. In women, the risk is 1.6 times higher than in men (4). The results of a systematic review illustrated that compared to other parts of the world, the incidence of SAH in Japan (22.7\%) and Finland (19.7\%) was higher, but lower in Central and South America (5).

According to the literature, risk factors for SAH are as follows: smoking, gender, advanced age, diabetes, obesity, inactivity, high cholesterol, high sodium diet, alcohol consumption, atrial fibrillation, familial history, and use of OCP medication (6-8). Another significant risk factor is hypertension, which is the most common and preventable cause of stroke (8).

The results of mortality and permanent disability due to SAH are relatively high. The results of a large retrospective study in Norway, conducted from 1984 to 2007, showed that the 30-day mortality rate in patients with SAH was $36 \%$ (6). While the Aneurysmal SAH mortality rate is 50\% and less than $60 \%$ of survivors returning to functional independence $(6,9)$.

\section{Objectives}

Given that the frequency and risk factors for SAH may vary in patients from different world regions, as well as 
those with and without an aneurysm, this study was conducted with the aim of determining the risk factors and outcome of SAH in patients with and without an aneurysm.

\section{Methods}

The present study is a cross-sectional (descriptiveanalytical) study that was performed on hospitalized SAH patients at Farshchian Hospital in Hamadan from 2015 to 2016. By using the Medical Records Department of the hospital, data on the hospitalized SAH patients were retrieved from medical records and entered the researcher-made checklist. The checklist included demographic information such as age and gender as well as clinical patient information like the history of hypertension, diabetes, hyperlipidemia, smoking, and disease outcome. Patients with ischemic stroke, hemorrhage in other parts of the brain and those with incomplete records were excluded. Descriptive statistics, including relative frequency, percentage, mean and standard deviation, were used to describe the study population. A chi-square test was used to compare the outcome of patients with and without an aneurysm. Data was analyzed using SPSS software, version 21 . The significance threshold was set at less than 0.05 .

\section{Results}

Overall, 69 patient records were investigated in this study. Among them 17 (24.63\%) were aneurysm. 46.38\% of patients were 60 years of age or older. There was a significant difference between the age of aneurysmal and nonaneurysmal in SAH patients; specifically, the proportion of aneurysmal SAH patients above 60 years of age was $11.76 \%$ compared to $57.69 \%$ in non-aneurysmal SAH patients $(\mathrm{P}=$ $0.003)$. Also, 40 (57.97\%) of the patients were male. A portion of the patient population had a history of risk factors for the disease, including 59.4\% with hypertension, $17.4 \%$ with diabetes, and $29 \%$ were smokers. However, as noted in Table 1, there was no significant correlation between these risk factors and aneurysm status of patients $(\mathrm{P}>0.05)$.

The outcomes of SAH in hospitalized patients are shown in Table 2. As indicated, the proportion of death, persistent defect, hydrocephaly and re-bleeding in the patients was $36.23 \%, 43.48 \%, 26.09 \%$, and $17.39 \%$, respectively. The different outcomes between SAH patients with and without aneurysm were not statistically significant $(\mathrm{P}>$ 0.05).

Of the 17 patients with aneurysm, the mean and standard deviation of aneurysm length and width were $7.71 \pm$ 10.40 and $5.33 \pm 7.60 \mathrm{~mm}$, respectively. As indicated in Table 3, the most common hemorrhage sites in aneurysmal SAH patients were ACOM (38.10\%) and MCA (28.57\%).

\section{Discussion}

The results of this study showed that the majority of SAH patients had over 60 years old and among them, non-aneurysmal SAH patients had a higher proportion of advanced age. Hypertension and smoking were two common preventable risk factors in aneurysmal and nonaneurysmal SAH patients. The disease led to death in approximately one-third of patients, and of the rest, nearly half had persistent complications.

In this study, the ratio of male patients was higher. In a study conducted by Eden et al. (10), the occurrence of SAH was higher in men than women. Whereas, in the study by Korja et al. (11) there was a significant relationship between female patients and the incidence of SAH. It is important to note that the incidence of SAH is more common in men; however, the greater life expectancy of women, coupled with the fact that the prevalence of stroke increases with age, results in higher frequency of stroke in women compared with men (12). Perhaps another reason for the high prevalence of this disease in women can be attributed to the effect of hormones and oral contraceptives in women (8).

In the present study, a significant percentage of patients had a history of hypertension, diabetes and smoking. In the study by Korja et al. (11), there was a significant relationship between patient history of smoking and systolic blood pressure with the incidence of SAH. In the review study conducted by Fejin et al. (13), the odds ratio of the incidence of SAH was 2.9 in smokers and 2.6 in hypertensive patients; in addition, the authors noted that smoking and hypertension were risk factors that had a significant relationship with the incidence of SAH. However, there was no significant relationship between diabetes and hypercholesterolemia with SAH. Conversely, in a study conducted by Lindbohm et al. (14), high cholesterol in men was a risk factor for SAH. In their study, Duran et al. (15), also noted that $50 \%$ of patients had a history of hypertension and $7.5 \%$ were smokers. Although in the present study, the proportion of patients who smoked and had hypertension was higher than the findings of Duran et al. (15).

In the present study on the outcomes of SAH in hospitalized patients, $36.2 \%$ of patients died and $43.5 \%$ had stable complication. The results of the study by Duran et al. (15) showed that in patients with SAH low systolic blood pressure at the time of admission was associated with a poor prognosis (15).

In some studies, they have reported a significant correlation between the size of aneurysms that are $7 \mathrm{~mm}$ or more and the incidence of SAH (11). A high aneurysm size is also associated with an acute physiological condition (10). In the present study, the average length and width of the aneurysm observed was over $7 \mathrm{~mm}$, but the outcome of the 


\begin{tabular}{|c|c|c|c|c|}
\hline Variable & Aneurysmal SAH $(\mathrm{N}=17)$ & Non-Aneurysmal SAH $(\mathrm{N}=52)$ & Total $(N=69)$ & P Value \\
\hline Age group & & & & 0.003 \\
\hline $20-29$ & 0 & $2(3.85)$ & $2(2.9)$ & \\
\hline $30-39$ & $1(5.88)$ & $5(9.62)$ & $6(8.7)$ & \\
\hline $40-49$ & $6(35.29)$ & $5(9.62)$ & $11(15.94)$ & \\
\hline $50-59$ & $8(47.06)$ & $10(19.23)$ & $18(26.09)$ & \\
\hline$\geq 60$ & $2(11.76)$ & $30(57.69)$ & $32(46.38)$ & \\
\hline Gender & & & & 0.52 \\
\hline Male & $11(64.71)$ & $29(55.77)$ & $40(57.97)$ & \\
\hline Female & $6(35.29)$ & $23(44.23)$ & $29(42.03)$ & \\
\hline History of hypertension & & & & 0.42 \\
\hline Yes & $12(70.59)$ & $29(55.77)$ & $41(59.42)$ & \\
\hline No & $5(29.41)$ & $23(44.23)$ & $28(40.58)$ & \\
\hline History of diabetes & & & & 0.97 \\
\hline Yes & $3(17.65)$ & $9(17.31)$ & $12(17.4)$ & \\
\hline No & $14(82.35)$ & $43(82.69)$ & $57(82.61)$ & \\
\hline History of Hyperlepidemia & & & & 0.87 \\
\hline Yes & $1(8.33)$ & $4(7.02)$ & $5(7.25)$ & \\
\hline No & $11(91.67)$ & $53(92.98)$ & $64(92.75)$ & \\
\hline Smoking status & & & & 0.51 \\
\hline Yes & $6(65.29)$ & $14(26.92)$ & $20(29.00)$ & \\
\hline No & $11(64.71)$ & $38(73.08)$ & $49(71.00)$ & \\
\hline
\end{tabular}

${ }^{\mathrm{a}}$ Values are expressed as No. (\%).

\begin{tabular}{|c|c|c|c|c|}
\hline Outcome & Aneurysmal SAH & Non-Aneurysmal SAH & Total & P Value \\
\hline Death & $7(41.18)$ & $18(34.62)$ & $25(36.23)$ & 0.62 \\
\hline Stable impairment & $5(29.41)$ & $25(48.08)$ & $30(43.48)$ & 0.18 \\
\hline Hydrocephaly & $2(11.76)$ & $16(30.77)$ & $18(26.09)$ & 0.12 \\
\hline Re-bleeding & $1(5.88)$ & $11(21.15)$ & $12(17.39)$ & 0.15 \\
\hline
\end{tabular}

${ }^{\mathrm{a}}$ Values are expressed as No. (\%).

disease and physiological status of the patients were not included in the study variables or goals. A study conducted by Park et al. (16), investigated the site of aneurysms in 384 patients with SAH. In their study, they noted that the most common site of aneurysm occurrence was MCA (51.9\%), followed by ICA (23.6\%) and ACOM (20.6\%). The sample size of the present study was smaller than that of Park et al. (16). In regards to the site of hemorrhage, the results of our study were not consistent with the above findings, which may be due to the anatomical differences of cerebral vessels in different races.

The primary limitation of this study was the sample size. The secondary limitation concerns possible inaccuracies and incomplete documentation in patient medical records.

\subsection{Conclusions}

The results of this study showed that SAH was more common in men and in the sixth decade of life regardless of gender. In addition, the majority of patients had a history of underlying diseases. We found that approximately one-third of patients died and nearly half of were left with long-term difficulties. Hypertension and smoking were two common modifiable risk factors in 


\begin{tabular}{|l|l}
\hline Table 3. Frequency of Aneurysm Location in Patients with SAH & \\
\hline Location & Values \\
\hline Left ACA & $2(9.52)$ \\
\hline Place of plugging right MCA & $1(4.76)$ \\
\hline ACOM & $4(19.05)$ \\
\hline Place of detachment ACOM left MCA & $3(14.29)$ \\
\hline Nut A3 & $1(4.76)$ \\
\hline Ophthalmic left carotid artery & $1(4.76)$ \\
\hline The location of the arteries top besila apcom & $2(9.52)$ \\
\hline Proximal MCA & $1(4.76)$ \\
\hline Interiors ACOM & $1(4.76)$ \\
\hline Place of plugging LCA (ACA an MCA) & $1(4.76)$ \\
\hline Bifurcation site right MCA & $1(4.76)$ \\
\hline Carotid sinus & $1(4.76)$ \\
\hline Proximal branch of fufani left MCA & $2(9.52)$ \\
\hline
\end{tabular}

${ }^{\mathrm{a}}$ Values are expressed as No. (\%).

aneurysmal and non-aneurysmal SAH patients. Unlike non-aneurysmal SAH patients, aneurysmal SAH was more common in younger patients.

\section{Acknowledgments}

The authors wish to thank the Faculty of Medicine as well as the Research and Technology Department of Hamadan University of Medical Sciences for approving this research study.

\section{Footnotes}

Authors' Contribution: MK and PB were responsible for the study concept and design. AM and SM were responsible for data analysis and interpretation. AM was solely responsible for statistical analysis. All authors were involved in drafting and critical revisions of the manuscript for important intellectual content.

Conflict of Interests: The authors do not report any conflict of interest.

Ethical Approval: The Ethics Committee at Hamadan University of Medical Sciences approved this study.

Funding/Support: This study was supported by the Research and Technology Department of Hamadan University of Medical Sciences.

\section{References}

1. Diao K, Lobos EA, Yirmibesoglu E, Basak R, Hendrix LH, Barbosa B, et al. Patient-reported quality of life during definitive and postprostatectomy image-guided radiation therapy for prostate cancer. Practical radiation oncology. 2017;7(2):e117-24. [PubMed: 28274402].

2. Barry C, Turner RJ, Corrigan F, Vink R. New therapeutic approaches to subarachnoid hemorrhage. Expert opinion on investigational drugs. 2012;21(6):845-59. [PubMed: 22530758].

3. Lanzino G, Kassell NF, Germanson TP, Kongable GL, Truskowski LL, Torner JC, et al. Age and outcome after aneurysmal subarachnoid hemorrhage: why do older patients fare worse? Journal of neurosurgery. 1996;85(3):410-8. [PubMed: 8751625].

4. Linn FHH, Rinkel GJE, Algra A, Van Gijn J. Incidence of subarachnoid hemorrhage: role of region, year, and rate of computed tomography: a meta-analysis. Stroke.1996;27(4):625-9. [PubMed: 8614919].

5. de Rooij NK, Linn FH, van der Plas JA, Algra A, Rinkel GJ. Incidence of subarachnoid haemorrhage: a systematic review with emphasis on region, age, gender and time trends. Journal of Neurology, Neurosurgery \& Psychiatry. 2007;78(12):1365-72. [PubMed: 17470467].

6. Sandvei MS, Mathiesen EB, Vatten LJ, Müller TB, Lindekleiv H, Ingebrigtsen $\mathrm{T}$, et al. Incidence and mortality of aneurysmal subarachnoid hemorrhage in two Norwegian cohorts, 1984-2007. Neurology. 2011;77(20):1833-9. doi: 10.1212/WNL.ob013e3182377de3. [PubMed: 22049205].

7. Johnson M, Bakas T. A review of barriers to thrombolytic therapy: implications for nursing care in the emergency department. Journal of Neuroscience Nursing. 2010;42(2):88-94. doi: 10.1097/JNN.ob013e3181ce5a48. [PubMed: 20422794].

8. Grysiewicz RA, Thomas K, Pandey DK. Epidemiology of ischemic and hemorrhagic stroke: incidence, prevalence, mortality, and risk factors. Neurologic clinics. 2008;26(4):871-95. doi: 10.1016/j.ncl.2008.07.003. [PubMed: 19026895].

9. Zacharia BE, Hickman ZL, Grobelny BT, DeRosa P, Kotchetkov I, Ducruet AF, et al. Epidemiology of aneurysmal subarachnoid hemorrhage. Neurosurgery Clinics. 2010;21(2):221-33. [PubMed: 20380965].

10. Eden SV, Meurer WJ, Sanchez BN, Lisabeth LD, Smith MA, Brown DL, et al. Gender and ethnic differences in subarachnoid hemorrhage. Neurology. 2008;71(10):731-5.

11. Korja $M$, Lehto $H$, Juvela $S$. Lifelong rupture risk of intracranial aneurysms depends on risk factors: a prospective Finnish cohort study. Stroke. 2014;45(7):1958-63. doi: 10.1161/STROKEAHA.114.005318.

12. Appelros P, Stegmayr B, Terént A. Sex differences in stroke epidemiology: a systematic review. Stroke. 2009;40(4):1082-90. doi: 10.1161/STROKEAHA.108.540781.

13. Feigin VL, Rinkel GJ, Lawes CM, Algra A, Bennett DA, van Gijn J, et al Risk factors for subarachnoid hemorrhage: an updated systematic review of epidemiological studies. Stroke. 2005;36(12):2773-80. doi: 10.1161/01.STR.0000190838.02954.e8. [PubMed: 16282541].

14. Lindbohm JV, Kaprio J, Korja M. Cholesterol as a risk factor for subarachnoid hemorrhage: a systematic review. PLoS One. 2016;11(4). doi: 10.1371/journal.pone.0152568. [PubMed: 27077917].

15. Duran L, Balci K, Kati C, Akdemir HU, Kocabicak E, Doğruel C. Has admission blood pressure any prognostic value in patients with subarachnoid hemorrhage: an emergency department experience. The Journal of Clinical Hypertension. 2013;15(10):737-41. doi: 10.1111/jch.12177. [PubMed: 24088282].

16. Park J, Cho J, Goh D, Kang D, Hamm I. Postoperative subdural hygroma and chronic subdural hematoma after unruptured aneurysm surgery: age, sex, and aneurysm location as independent risk factors. Journal of neurosurgery. 2016;124(2):310-7. doi: 10.3171/2015.1.JNS14309. [PubMed: 26275003]. 